\title{
Correction to: Genetic differentiation in the mountainous star coral Orbicella faveolata around Cuba
}

\author{
Gabriela Ulmo-Díaz ${ }^{1}$ Didier Casane ${ }^{2,3}$ - Louis Bernatchez ${ }^{4}$ Patricia González-Díaz $^{1}$ • \\ Amy Apprill ${ }^{5} \cdot$ Jessy Castellanos-Gell $^{1} \cdot$ Leslie Hernández-Fernández $^{6}$. \\ Erik García-Machado ${ }^{1}$
}

\section{Correction to: Coral Reefs https://doi.org/10.1007/s00338-018-1722-x}

Since the publication of this paper, the authors have been made aware of an error in Table 1 that does not affect the conclusions reached or any other part of the paper. Namely, the last two columns in Table $1\left(\mathrm{H}_{\mathrm{o}} \pm \mathrm{SD}\right.$ and $\left.\mathrm{uH}_{\mathrm{e}} \pm \mathrm{SD}\right)$ are inversed. Table 1 should be as follows:

We deeply apologize for any inconvenience this may have caused.

The original article can be found online at https://

doi.org/10.1007/s00338-018-1722-x.

Gabriela Ulmo-Díaz

gabriela.ulmo-diaz.1@ulaval.ca

1 Centro de Investigaciones Marinas (CIM-UH), Universidad de La Habana, Calle 16, No. 114 entre 1ra y 3ra, Miramar, 11300 Playa, La Habana, Cuba

2 Laboratoire ÉvolutionGénomesComportementÉcologie, CNRS Université Paris-Sud UMR 9191, IRD UMR 247, Avenue de la Terrasse, Bâtiment 13, boîte postale 1, 91198 Gif-sur-Yvette, France

3 Sorbonne Paris-Cité, Université Paris-Diderot, Paris, France

4 Institut de Biologie Intégrative Et Des Systèmes (IBIS), Pavillon Charles-Eugène-Marchand Université Laval, Quebec, QC G1V 0A6, Canada

5 Woods Hole Oceanographic Institution, 266 Woods Hole Road, Woods Hole, MA 02543, USA

6 Centro de Investigaciones de Ecosistemas Costeros (CIEC), 69400 Cayo Coco, Morón, Ciego de Ávila, Cuba 
Table 1 Genetic diversity estimates of Orbicella faveolata in Cuba

\begin{tabular}{|c|c|c|c|c|c|c|c|}
\hline \multirow[t]{2}{*}{ Locality } & \multicolumn{3}{|c|}{ mtDNA } & \multicolumn{4}{|c|}{ Microsatellite loci } \\
\hline & $\mathrm{Hn}$ & $h \pm \mathrm{SD}$ & $\pi \pm \mathrm{SD}$ & Allelic richness & Unique alleles & $\mathrm{H}_{\mathrm{o}} \pm \mathrm{SD}$ & $\mathrm{uH}_{\mathrm{e}} \pm \mathrm{SD}$ \\
\hline Coco Key & 7 & $0.911 \pm 0.077$ & $0.00186 \pm 0.00036$ & 5.49 & 4 & $0.721 \pm 0.112$ & $0.856 \pm 0.059$ \\
\hline Baracoa Beach & 5 & $0.667 \pm 0.113$ & $0.00101 \pm 0.00023$ & 4.96 & 10 & $0.729 \pm 0.126$ & $0.812 \pm 0.067$ \\
\hline Los Colorados & 11 & $0.780 \pm 0.078$ & $0.00192 \pm 0.00038$ & 5.24 & 13 & $0.739 \pm 0.101$ & $0.807 \pm 0.089$ \\
\hline Los Canarreos & 5 & $0.647 \pm 0.060$ & $0.00126 \pm 0.00034$ & 4.65 & 5 & $0.733 \pm 0.081$ & $0.788 \pm 0.063$ \\
\hline Jardines de la Reina & 7 & $0.777 \pm 0.050$ & $0.00122 \pm 0.00014$ & 4.79 & 5 & $0.759 \pm 0.089$ & $0.798 \pm 0.06$ \\
\hline
\end{tabular}

Hn: haplotype number. $h$ : haplotype diversity. $\pi$ : nucleotide diversity. $\mathrm{H}_{\mathrm{o}}$ : Observed heterozygosity. $\mathrm{uH}_{\mathrm{e}}$ : unbiased expected heterozygosity. $\mathrm{SD}$ : standard deviation

Publisher's Note Springer Nature remains neutral with regard to jurisdictional claims in published maps and institutional affiliations. 